\title{
Time-Space Scale Effects in Numerically Computing Flowfields and a New Approach to Numerical Flow Simulation
}

\author{
Z. Gao ${ }^{1}$ and F.G. Zhuang ${ }^{2}$ \\ ${ }^{1}$ Institute of Mechanics, Chinese Academy of Sciences \\ Beijing 100080, China \\ ${ }^{2}$ Science and Technology Committee of China Aerospace Corporation \\ Beijing 100830, China
}

\begin{abstract}
Numerical solutions of the large scale (LS) average equations and small scale (SS) interacting equations describing fluid flow presented by the authors are conducted for two-dimensional channel flow with multiscale structure. A pseudospectral method is used. In the range of Reynolds number from $10^{2}$ to $3 \times 10^{3}$, some important effects of nonlinear interaction between the large scale and small scale eddies are shown. Comparison of the numerical solutions of LS- and SS-equations with those of NS equations is also given.
\end{abstract}

\section{Introduction}

In normal cases of computing numerically flowfields of scientific and engineering interest, one needs to solve the Navier-Stokes(NS) equations in its laminar flow region and to assume the transitional region from laminar to turbulent flow and to solve the Reynolds equations or the Large Eddy Simulation(LES) equations with the aid of turbulence models in its turbulent flow region ${ }^{[1]}$. It is obviously difficult to make a guess at transitional region in complex three-dimensional flows and vortex flows. On the other hand, direct numerical simulations of the NS equations(DNS), i.e., computing numerically NS equations at the smallest time-space scale $\left(\Delta t_{N}, \Delta_{N}\right)$ level, can predict the evolution of all significant scales of the flow without distinguishing between laminar-transition-turbulent flows as well as using any turbulence models ${ }^{[2]}$. The above-stated puzzled situation arises because a complex flow containing partly turbulent flow region contains a broad time-space scale range and there exist nonlinar interactions between large and small eddies with relatively large time-space scales $\left(\Delta t_{n}, \Delta_{i, n}\right)$, where

$$
\Delta t_{N} \ll \Delta t_{n} \ll \frac{L}{U}, \quad \Delta_{N} \ll \Delta_{i, n} \ll L \quad(i=1,2,3)
$$

$U$ and $L$ are the characteristic flow speed and length, respectively. However, NS equations do not contain terms describing interaction between relatively large scale eddies. Our qualitative and quantitative knowledge for complex flows depends on time-space scales $\Delta t_{n}$ and $\Delta_{i, n}(i=1,2,3)$ selected in measuring or computing the 
flows. Therefore, from the theoretical viewpoint, it would be valuable to develop new equations governing fluid flow and corresponding numerical methods. The new equations can be used at the smallest and relatively large time-space scales and can describe nonlinear interaction between large and small scale eddies and would be simultaneously suitable to laminar-transition-turbulent flows ${ }^{[3]}$.

\section{Basic Equations}

The basic equations presented by the authors are composed of the large scale(LS) average equations governing the large scale average motion of the flow and the small scale(SS) interacting equations governing the small scale (or edding) motion. For incompressible flows, the LS-and SS-equations are respectively

$$
\begin{aligned}
& \frac{\partial \bar{u}_{i}}{\partial x_{i}}=0 \\
& \frac{\partial \bar{u}_{i}}{\partial t}+\frac{\partial}{\partial x_{j}}\left(\bar{u}_{i} \cdot \bar{u}_{j}\right)=-\frac{1}{\rho} \frac{\partial \bar{p}}{\partial x_{i}}+\nu \frac{\partial^{2} \bar{u}_{i}}{\partial x_{j} \partial x_{j}}-\frac{\partial}{\partial x_{j}}\left(\bar{\lambda}_{i j}\right) \\
& \frac{\partial}{\partial x_{i}}\left(u_{i}-\bar{u}_{i}\right)=0 \\
& \frac{\partial}{\partial t}\left(u_{i}-\bar{u}_{i}\right)+\frac{\partial}{\partial x_{j}}\left[\left(u_{i}-\bar{u}_{i}\right)\left(u_{j}-\bar{u}_{j}\right)\right]=-\frac{1}{\rho} \frac{\partial(p-\bar{p})}{\partial x_{i}}+\nu \frac{\partial^{2}\left(u_{i}-\bar{u}_{i}\right)}{\partial x_{j} \partial x_{j}} \\
& \quad-\frac{\partial}{\partial x_{j}}\left(\bar{u}_{i} \bar{u}_{j}-\overline{\bar{u}}_{i} \bar{u}_{j}\right)-\frac{\partial}{\partial x_{j}}\left[\bar{u}_{i}\left(u_{j}-\bar{u}_{j}\right)+\bar{u}_{j}\left(u_{i}-\bar{u}_{i}\right)\right]+\frac{\partial \bar{\lambda}_{i j}}{\partial x_{j}} \\
& (i=1,2,3)
\end{aligned}
$$

where

$$
\begin{aligned}
& \left(\bar{u}_{i}, \bar{p}, \overline{\bar{u}_{i} \bar{u}_{j}}, \lambda_{i j}\right)= \\
& \frac{1}{\Delta t_{n} \prod_{k}^{3} \Delta_{k, n}} \int_{t}^{t+\Delta t_{n}} \iint_{\substack{x_{k}-\frac{1}{2} \Delta_{k, n} \\
t_{k}}}^{x_{k}+\frac{1}{2} \Delta_{k, n}}\left\{u_{i}, p, \bar{u}_{i} \cdot \bar{u}_{j},\left[\left(u_{i}-\bar{u}_{i}\right)\left(u_{j}-\bar{u}_{j}\right)+\bar{u}_{i}\left(u_{j}-\bar{u}_{j}\right)\right]\right\} d \xi_{k} d \tau
\end{aligned}
$$

$u_{i}(i=1,2,3)$ and $p$ are the flow speed components and the pressure, respectively. $\Delta t_{n}$ and $\Delta_{i, n}(i=1,2,3)$ are the time and space scales selected in computing the flow; we call them cutoff scales. The second term of the right hand of the equations $(2.1 \mathrm{~b})$ is the molecular viscous term. $\frac{\partial}{\partial x_{j}}\left(\bar{\lambda}_{i j}\right)$ are called the eddy viscous terms, which express the nonlinear interaction between the eddies with scales smaller and larger than the cutoff scales. The fourth term of the right hand of the equations $(2.2 \mathrm{~b})$ is the remote action term of the eddies with scales larger than the cutoff scales on the small scale eddies.

The main properties and functions of the LS-and SS-equations are as follows. (1) When the cutoff time-space scales are small enough, or they are much smaller than the intrinsic physical scales of the flows, such as the cases of low Reynolds number laminar flows, the LS-equations reduce to the Navier-Stokes equations, while the SS-equations 
can be cast away because they give zero solutions $\left(u_{i}-\bar{u}_{i}\right) \cong 0(i=1,2,3)$ and $(p-\bar{p}) \cong 0$. (2) For turbulent flows, if only the cutoff time scale is small enough, the LS-equations are equivalent to the Large Eddy Simulation (LES) equations; on the other hand, if only the cutoff space scales are small enough, and let $\overline{\bar{u}}_{i} \cdot \bar{u}_{j}=\bar{u}_{i} \cdot \bar{u}_{j}$, the LS-equations are equivalent to the Reynolds equations; under the above two circumstances, the SS-equations determine the small scale motion of the flow and also close the LSequations. (3) The LS-and SS-equations open a new way to compute large and small scale eddies as well as the nonlinear interaction between different scale eddies of the flow, which may be turbulent or transitional or laminar, such as the laminar bifurcation phenomenon caused by multiple-vortex secondary flow. (4) Using LSand SS-equations we can compute in a unified manner laminar-transition-turbulent parts of the complex flow without supposing transitional regions and distinguishing between laminar and turbulent flows and using any turbulence models. Hence the solution of LS- and SS-equations links organically direct numerical simulation at the smallest scale level with the normal numerical computation at relatively large scale level. Especially, the solving of the SS-equations can be confined within certain local regions of the flowfield and lighten greatly the burden on computer. In a sense, the LSand SS-equations open a relatively realistic way to compute high Reynolds number complex flows.

\section{$3 \quad$ Numerical Method}

We use a pseudospectral method to solve simultaneously LS- and SS-equations for two-dimensional channel flow with multiscale structure. Because only two first-order partial derivative terms are added into the SS-equations (2.2) compared with the LSequations (2.1), the SS-equations (2.2) and LS-equations (2.1) can be solved using the same algorithm. Two discretizations, Fourier collocation and Chebyshev collocation, are selected in the streamwise and normal directions, respectively. We adopt periodic boundary conditions and uniform grids in the streamwise direction and the Chebyshev collocation (grids) distribution and the boundary condition of impermeable and no-slip in the normal direction, respectively. The finest and the coarsest grids are $128 \times 128$ and $8 \times 8$, respectively. The time-stepping is a second-order semi-implicit scheme with the time-step chosen so small that the spatial errors predominate.

\section{Analysis and Discussion}

The two-dimensional disturbances are added to initial flow.

$$
\begin{aligned}
& u=u_{0}=1-y^{n}+\varepsilon \frac{\pi}{k_{1}} \cos k_{1} x \sin \left[\left(2 k_{2}+1\right) \pi y\right] \\
& v=v_{0}=-\varepsilon \frac{\sin k_{1} x}{\left(2 k_{2}+1\right)}\left\{1+\cos \left[\left(2 k_{2}+1\right) \pi y\right]\right\}
\end{aligned}
$$

where $n=2$ or 8 , the amplitude parmeter $\varepsilon$ of the initial disturbance is about $10^{-4}$ to 0.1 and the "wave number" $k$ is about 1 to 80 . The initial velocities satisfy the 
continuity equation. The evolution of all significant scales and the important influences of the eddying motion on the average variables $\bar{u}_{i}(i=1,2,3)$ and $\bar{p}$ are given by solving simultaneously the LS- and SS-equations and compared with the corresponding numerical results of NS equations. Some main results are as follows. In all the cases of relatively low Re, the eddying motion decays with the time (see Fig.1 and 6). In the initial and intermediate stages the eddy viscous force, the nonlinear interaction between the large scale and small scale eddies, is larger than or comparable to the molecular viscous force (see Fig.2 and 3), Fig.4 and 5 show that in the middle stage of the evolution $(u-\bar{u})$ and $(v-\bar{v})$ become larger than their initial values; this is because the small scale eddies are excited by the large scale average motion. The differences between the numberical solutions of LS- and SS-equations and NS equations are remarkable (also see Table 1). In the later stage the eddying motion disappears and the solution of LS-equations becomes the same as that of NS equations. In the cases of relatively large Re, the small scale eddies do not roughly decay (see Fig.6), and the numerical solutions of both the LS- and SS-equations and NS equations are found to be sensitive to the initial disturbances and grid refinement. The critical Reynolds number at which the disturbances no longer decay is predicted numerically. The above statements agree with the theoretical inference for the mathematical properties of LS- and SS-equations and also with other study results such as $^{[4,5]}$ :

\section{References}

[1] D.A.Anderson, et al "Computational Fluid Mechanics and Heat Transfer"

[2] (Hemisphere/McGraw-Hill, New York, 1984).

[3] W.C.Reynolds "Whither Turbulence" Cornell University (1989)

[4] Z.Gao "A New Conservation Equations of Fluid Dynamics" IMCAS STR-92026, 1992.

[5] R.D.Moser, P.Moin, A.Leonerd, Jour.Comput.Phys. Vol.52, P524 544, 1983.

[6] L.Lleiser, Schumann, Proc. of the 3rd GAMM-Conf. on Num. Meth. in fluid Mech. P165 173, 1980. 

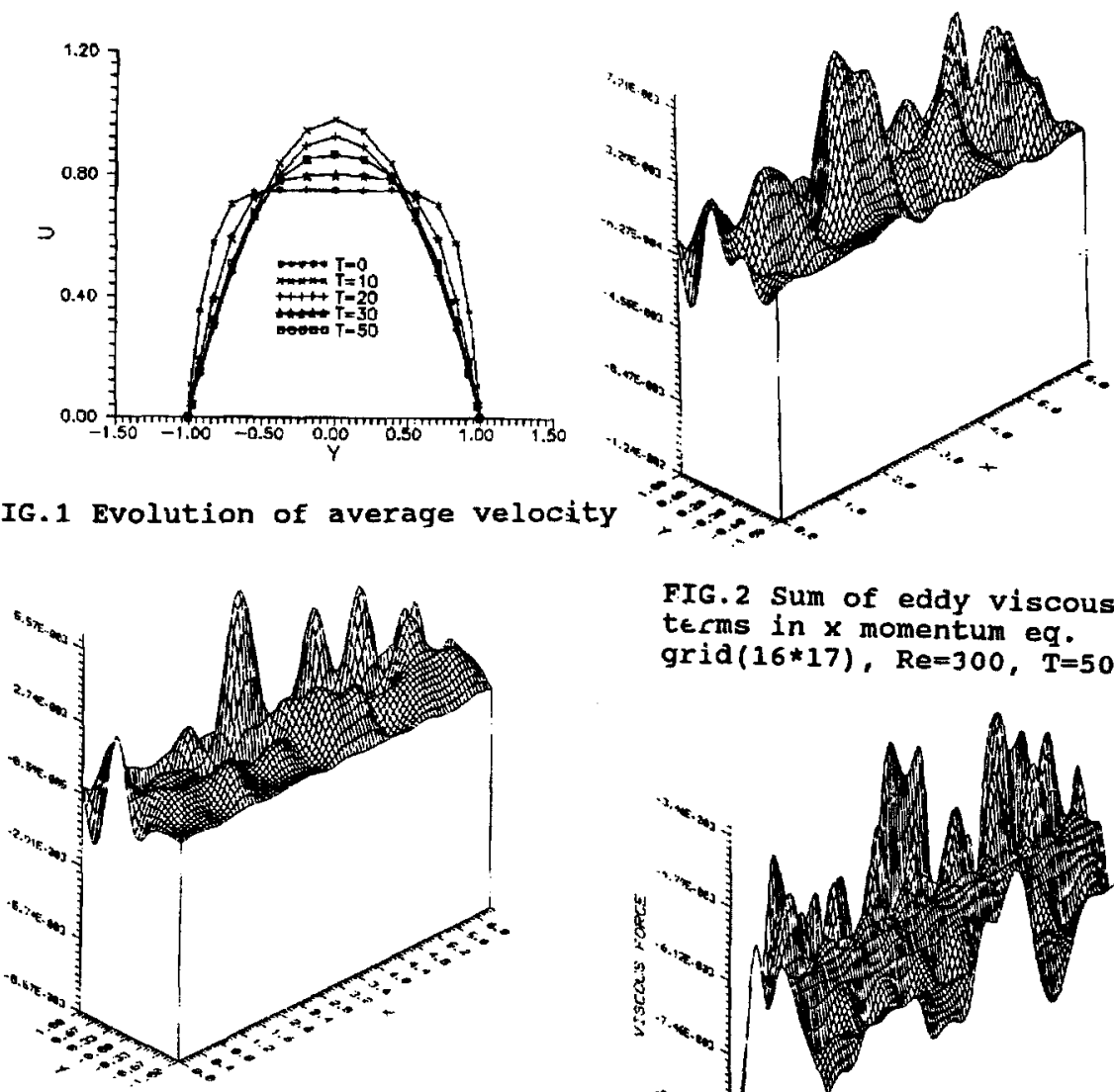

FIG.2 Sum of eddy viscous terms in $x$ momentum eq. grid $(16 * 17), \quad R e=300, T=50$

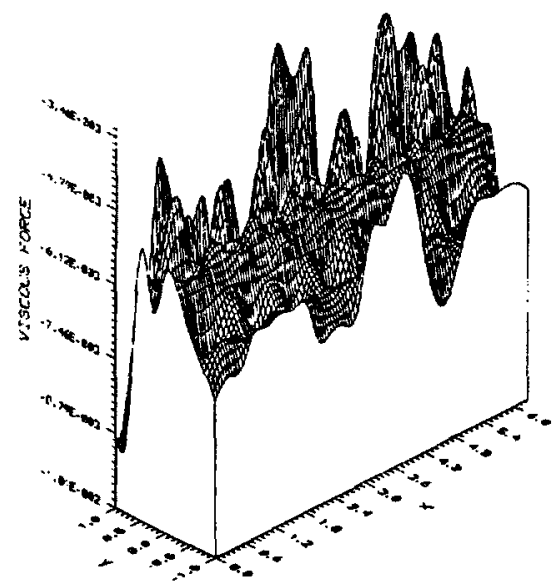

FIG.3 Sum of eddy viscous terms in $y$ momentum eq. grid $(16 * 17), \quad R e=300, T=50$

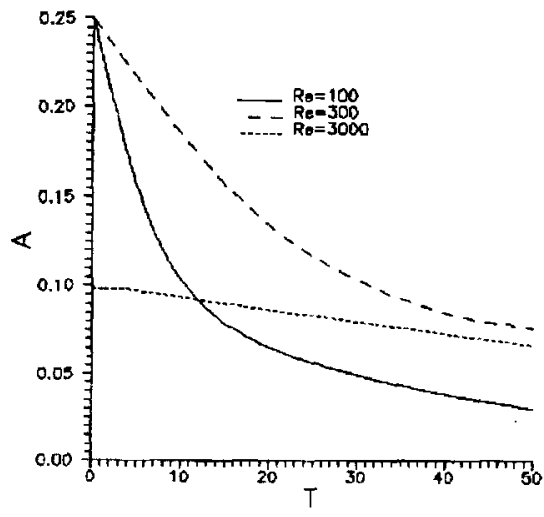

FIG.4 Sum of viscous terms in $x$ momentum eq. grid $(16 * 17), \quad \operatorname{Re}=300, T=50$

FIG.6 Evolution of small scale amplitude 

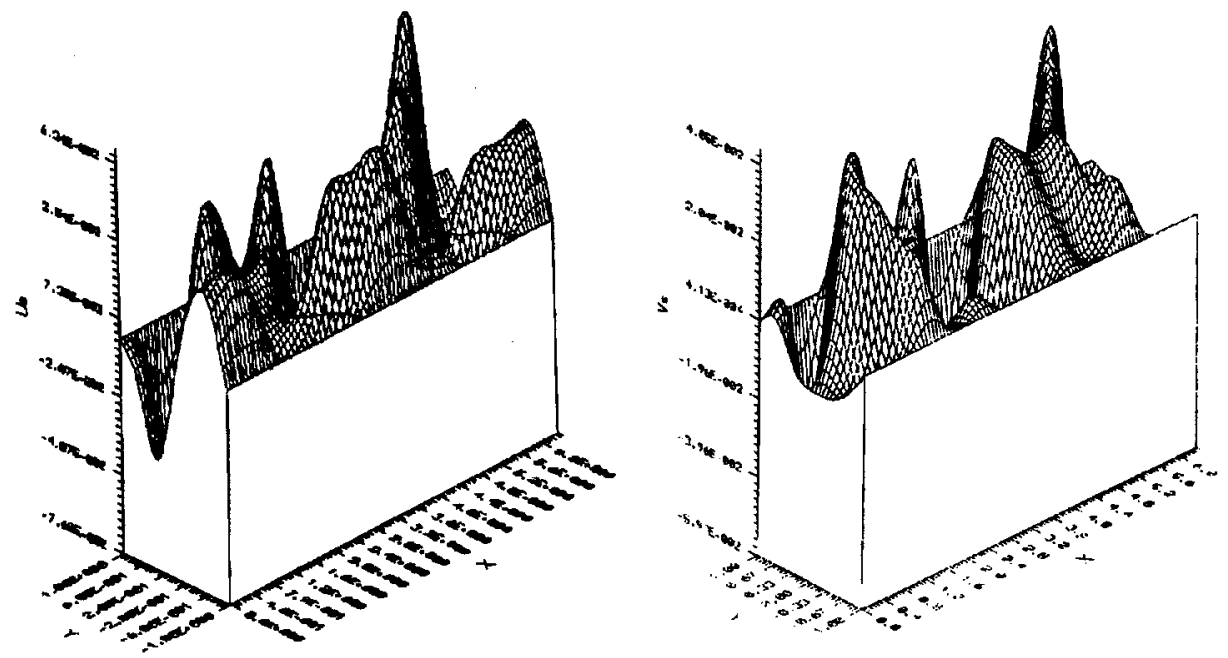

FIG.5 Small scale velocity $\operatorname{grid}(64 * 65), \quad R e=300, T=50$

\begin{tabular}{|c|c|c|}
\hline \multirow{2}{*}{$\mathbf{Y}$} & \multicolumn{2}{|c|}{0} \\
\hline & GZ. EQ. & . NS EQ. \\
\hline $\begin{array}{l}-0.100000 \mathrm{E}+01 \\
-0.980785 \mathrm{E}+00 \\
-0.923880 \mathrm{E}+00 \\
-0.831470 \mathrm{E}+00 \\
-0.707107 \mathrm{E}+00 \\
-0.555570 \mathrm{E}+00 \\
-0.382683 \mathrm{E}+00 \\
-0.195090 \mathrm{E}+00 \\
0.349148 \mathrm{E}-14 \\
0.195090 \mathrm{E}+00 \\
0.382683 \mathrm{E}+00 \\
0.555570 \mathrm{E}+00 \\
0.707107 \mathrm{E}+00 \\
0.831470 \mathrm{E}+00 \\
0.923880 \mathrm{E}+00 \\
0.980785 \mathrm{E}+00 \\
0.100000 \mathrm{E}+01\end{array}$ & $\begin{array}{r}-0.167711 \mathrm{E}-15 \\
0.355285 \mathrm{E}-01 \\
0.137050 \mathrm{E}+00 \\
0.290095 \mathrm{E}+00 \\
0.469581 \mathrm{E}+00 \\
0.646310 \mathrm{E}+00 \\
0.793752 \mathrm{E}+00 \\
0.888307 \mathrm{E}+00 \\
0.922699 \mathrm{E}+00 \\
0.893403 \mathrm{E}+00 \\
0.798655 \mathrm{E}+00 \\
0.655533 \mathrm{E}+00 \\
0.489344 \mathrm{E}+00 \\
0.310566 \mathrm{E}+00 \\
0.147432 \mathrm{E}+00 \\
0.379175 \mathrm{E}-01 \\
-0.182892 \mathrm{E}-15\end{array}$ & $\begin{array}{r}-0.955116 \mathrm{E}-16 \\
0.364834 \mathrm{E}-01 \\
0.140180 \mathrm{E}+00 \\
0.294611 \mathrm{E}+00 \\
0.474946 \mathrm{E}+00 \\
0.652123 \mathrm{E}+00 \\
0.798562 \mathrm{E}+00 \\
0.893743 \mathrm{E}+00 \\
0.926544 \mathrm{E}+00 \\
0.893745 \mathrm{E}+00 \\
0.798566 \mathrm{E}+00 \\
0.652127 \mathrm{E}+00 \\
0.474950 \mathrm{E}+00 \\
0.294613 \mathrm{E}+00 \\
0.140181 \mathrm{E}+00 \\
0.364837 \mathrm{E}-01 \\
-0.850801 \mathrm{E}-16\end{array}$ \\
\hline
\end{tabular}

Table 1. Distribution of velocity $U$ at $X=0.335103 E+01$ grid(16*17), $\operatorname{Re}=300, T=50$ 